\title{
Biomarkers to guide antibiotic timing and administration in infected patients presenting to the emergency department
}

\author{
Mari Rosenqvist ${ }^{1 *}$, Darius Cameron Wilson ${ }^{2}$, Lena Tegnér ${ }^{1}$, Maria Bengtsson-Toni ${ }^{1}$, Marjaneh Peyman ${ }^{1}$, \\ Juan Gonzalez del Castillo ${ }^{3}$, Kordo Saeed ${ }^{4}$ and Olle Melander ${ }^{5}$
}

Antibiotics are often prescribed in the emergency department (ED) to patients presenting with a suspected infection before any definitive diagnosis can be made [1]. However, increasing antibiotic resistance and detrimental effects on the microbiota require their use to be limited to those with a high likelihood of bacterial infection or the potential for further clinical deterioration. Conversely, withheld or delayed treatment in higher severity patients may lead to increased morbidity and mortality rates [2]. Thus, an accurate assessment of antibiotic requirement and speed of administration is crucial.

Current tools to aid clinical decision-making include the use of procalcitonin (PCT) and C-reactive protein (CRP). However, recent interventional evidence in the ED has shown few differences between conventional biomarker-guided therapy and standard practice $[1$, $3]$, despite protocol compliance, patient selection and cut-off concerns. This post hoc analysis of a patient subset (Malmö, Sweden) from our previous investigation [4] compared the use of PCT, CRP and lactate to the novel biomarker mid-regional proadrenomedullin (MR-proADM) in guiding antibiotic administration during treatment within the ED.

Within this subset $(N=213), 26(12.2 \%)$, patients were prescribed antibiotics $<48 \mathrm{~h}$ prior to presentation, whilst 187 (87.8\%) were administered antibiotics during ED assessment. Of these patients, 164 (77.0\%) were treated with intravenous (i.v.) and $23(10.8 \%)$ with oral antibiotics. The median time to initial administration was 93 [28-160] min, with $71(43.8 \%)$ patients receiving therapy within $60 \mathrm{~min}$. Univariate and multivariate logistic regression found that MR-proADM had the strongest association with the requirement for antibiotic administration during ED treatment (Table 1). Interestingly, MR-proADM (Spearman $\rho=-0.31, p<$ 0.001 ) and lactate (Spearman $\rho=-0.25, p=0.002$ ) were the only parameters to be significantly negatively correlated with the time to antibiotic administration, with significant differences found at optimised MR-proADM cut-offs for antibiotic administration $(1.27 \mathrm{nmol} / \mathrm{L}: 139$ [76-211] vs 43 [26-135] $\mathrm{min} ; p<0.001)$ or pre-established [4] cut-offs for mortality prediction (1.54 nmol/L: 124 [33-199] vs 42 [26-122] min; $p=$ 0.002). Similar results were also found for MR-proADM within previously established PCT concentration ranges [5] (Table 2), with an absence of ICU admission or 28-day mortality in patients with low MR-proADM concentrations, despite lower antibiotic administration rates and a significantly longer time to administration.

Results suggest that delayed antibiotic administration in patients with low MR-proADM concentrations may result in few adverse effects, potentially allowing for a more detailed clinical assessment prior to any subsequent initiation. Further studies in larger patient populations are required to confirm these initial findings.

\footnotetext{
* Correspondence: mari.rosenqvist@med.lu.se

${ }^{1}$ Infectious Disease Unit, Skåne University Hospital, Department of Clinical

Sciences Malmö, Lund University, Malmö, Sweden

Full list of author information is available at the end of the article
} 
Table 1 Univariate and Multivariate analyses found that MR-proADM had the strongest correlation with the requirement for antibiotic administration during ED treatment

\begin{tabular}{lllllll}
\hline Biomarker & Patient population $(N)$ & Antibiotic administration $(N)$ & $p$ value & $C$ index & Univariate OR [95\% Cl] & Multivariate OR [95\% CI] \\
\hline MR-proADM & 213 & 164 & $<0.001$ & 0.76 & $3.1[1.9-4.9]$ & $3.3[1.9-5.9]$ \\
PCT & 213 & 164 & $<0.001$ & 0.74 & $2.7[1.7-4.3]$ & $2.7[1.7-4.5]$ \\
CRP & 207 & 159 & $<0.001$ & 0.68 & $1.8[1.3-2.5]$ & $1.9[1.4-2.8]$ \\
Lactate & 204 & 158 & 0.002 & 0.66 & $1.8[1.2-2.6]$ & $1.6[1.1-2.5]$ \\
\hline
\end{tabular}

Age, cardiovascular, neurological, renal and malignancy comorbidities were used as adjusting variables within the multivariate regression analysis, as previously outlined [4]. Univariate and multivariate odds ratios were expressed per $1 \mathrm{SD}$ increment of the log-transformed value for each respective biomarker. Cl confidence interval, CRP C-reactive protein, DF degrees of freedom, MR-proADM mid-regional proadrenomedullin, $N$ number, $O R$ odds ratio, $P C T$ procalcitonin

Table 2 Low MR-proADM concentrations resulted in an absence of ICU admission or 28-day mortality, despite lower antibiotic administration rates and a significantly longer time to administration, irrespective of corresponding PCT concentration

\begin{tabular}{|c|c|c|}
\hline \multirow[t]{2}{*}{ Patient subgroups } & \multicolumn{2}{|c|}{ MR-proADM concentration } \\
\hline & $<1.27(\mathrm{nmol} / \mathrm{L})$ & $\geq 1.27(\mathrm{nmol} / \mathrm{L})$ \\
\hline \multicolumn{3}{|l|}{ Subgroup 1: PCT concentration: < $0.25 \mu \mathrm{g} / \mathrm{L}(N=106)$} \\
\hline Patients $(N)$ & 65 & 41 \\
\hline Antibiotic administration $(N, \%)$ & $35(53.8 \%)$ & $34(82.9 \%)$ \\
\hline Time to antibiotic administration (min) (median, Q1-Q3) & $127[45.0-220]$ & $42[25.8-116]$ \\
\hline Composite of 28-day mortality and ICU admission $(N, \%)$ & $0(0.0 \%)$ & $7(17.1 \%)$ \\
\hline \multicolumn{3}{|l|}{ Subgroup 2: PCT concentration: $\geq 0.25$ and $<0.50 \mu \mathrm{g} / \mathrm{L}(\mathrm{N}=24)$} \\
\hline Patients $(N)$ & 8 & 16 \\
\hline Antibiotic administration $(N, \%)$ & $7(87.5 \%)$ & $15(93.8 \%)$ \\
\hline Time to antibiotic administration (min) (median, Q1-Q3) & $165[88-305]$ & 50 [19.3-186] \\
\hline Composite of 28-day mortality and ICU admission $(N, \%)$ & $0(0.0 \%)$ & $1(6.3 \%)$ \\
\hline \multicolumn{3}{|l|}{ Subgroup 3: PCT concentration: $\geq 0.50 \mu \mathrm{g} / \mathrm{L}(N=83)$} \\
\hline Patients $(N)$ & 21 & 62 \\
\hline Antibiotic administration $(N, \%)$ & $15(71.4 \%)$ & $59(95.2 \%)$ \\
\hline Time to antibiotic administration (min) (median, Q1-Q3) & $131[92.8-166]$ & $45[26-136.5]$ \\
\hline Composite of 28-day mortality and ICU admission ( $N, \%)$ & $0(0.0 \%)$ & $15(24.2 \%)$ \\
\hline
\end{tabular}

MR-proADM mid-regional proadrenomedullin, $N$ number, $P C T$ procalcitonin, $Q$ quartile

\section{Abbreviations}

Cl: Confidence interval; CRP: C-reactive protein; ED: Emergency department; i.v.: Intravenous; ICU: Intensive care unit; MR-proADM: Mid-regional proadrenomedullin; N: Number; OR: Odds ratio; PCT: Procalcitonin

\section{Acknowledgements}

The authors are grateful to the staff at Skåne University Hospital for their assistance in identifying eligible patients for enrollment.

\section{Funding}

For the purpose of this letter, no specific funding was received. Neither for the original study nor this letter did the funding organisation have any role in the design and conduct of the trial; collection, management, analysis, and interpretation of the data; preparation, review, or approval of the manuscript and decision to submit the manuscript for publication.

\section{Availability of data and materials}

The datasets used and/or analysed during the present study are available from the corresponding author upon reasonable request.

\section{Authors' contributions}

MR was the primary author and editor of the manuscript. OM was the principal investigator. MR, LT, MBT, MP and OM collected the study data and, in collaboration with DCW (up until January 2019), KS and JGdC, contributed to the evaluation and interpretation of the data as well as writing and editing of the manuscript. MR and OM performed the statistical analysis of the data. All authors critically reviewed and approved the final manuscript.

Ethics approval and consent to participate

The study was approved by the Regional Ethical Review Board at Lund University, Sweden (2013/635), and was conducted in accordance with the Helsinki Declaration. Informed consent was obtained from all patients or their next of kin.

\section{Consent for publication}

No individual participant data is reported that would require consent to publish from the participant (or legal parent or guardian for children).

\section{Competing interests}

All authors have provided information on potential conflicts of interests directly or indirectly related to the work submitted in the journal's disclosure forms. At the time of initial analysis, interpretation and writing, DCW was an employee of BRAHMS GmbH, which holds patent rights on the procalcitonin and mid-regional proadrenomedullin assay. All other authors declare that they have no competing interests. 


\section{Publisher's Note}

Springer Nature remains neutral with regard to jurisdictional claims in published maps and institutional affiliations.

\section{Author details}

'Infectious Disease Unit, Skåne University Hospital, Department of Clinical Sciences Malmö, Lund University, Malmö, Sweden. ${ }^{2}$ B.R.A.H.M.S GmbH, Hennigsdorf, Germany. ${ }^{3}$ Emergency Department, Instituto de Investigación Sanitaria (IdISSC), Hospital Clínico San Carlos, Madrid, Spain. ${ }^{4}$ Department of Microbiology, Hampshire Hospitals NHS Foundation Trust, Winchester and Basingstoke, UK. ${ }^{5}$ Department of Internal Medicine, Skåne University Hospital, Malmö, Sweden

Received: 26 March 2019 Accepted: 4 April 2019

Published online: 25 April 2019

References

1. van der Does $Y$, Limper M, Jie KE, Schuit SCE, Jansen $H$, Pernot $N$, et al. Procalcitonin-guided antibiotic therapy in patients with fever in a general emergency department population: a multicenter noninferiority randomized clinical trial (HiTEMP study). Clin Microbiol Infect. 2018;24(12):1281-9.

2. Seymour CW, Gesten F, Prescott HC, Friedrich ME, Iwashyna TJ, Phillips GS, et al. Time to treatment and mortality during mandated emergency care for sepsis. N Engl J Med. 2017;376:2235-44.

3. Huang DT, Yealy DM, Filbin MR, Brown AM, Chang CH, Doi Y, et al. Procalcitonin-guided use of antibiotics for lower respiratory tract infection. N Engl J Med. 2018;379:236-49

4. Saeed K, Wilson DC, Bloos F, Schuetz $P$, van der Does $Y$, Melander O, et al. The early identification of disease progression in patients with suspected infection presenting to the emergency department: a multi-centre derivation and validation study. Crit Care. 2019;23:40.

5. Schuetz P, Beishuizen A, Broyles M, Ferrer R, Gavazzi G, Gluck EH, et al Procalcitonin (PCT)-guided antibiotic stewardship: an international experts consensus on optimized clinical use. Clin Chem Lab Med. 2019. https://doi. org/10.1515/cclm-20181181 Epub ahead of print 